\title{
Mise en série et tradition de problèmes dans les arithmétiques en langue vulgaire de la fin du Moyen Âge
}

\author{
Stéphane Lamasséa \\ LAMOP, Université Paris 1, Centre Sorbonne, 17 rue de la Sorbonne, Paris $5^{\mathrm{e}}$ Labex HASTEC \\ ANR-10-LABX-85, France
}

\begin{abstract}
Résumé. Cet article se propose de dresser le bilan d'une étude à présent ancienne sur les problèmes qui sont véhiculés au $\mathrm{XV}^{\mathrm{e}}$ siècle au travers d'un corpus arithmétiques en langue vernaculaire. Il s'agit, ici, essentiellement d'une présentation de la méthode utilisée alors pour pister les héritages entre les problèmes. La démarche informatique nous a conduits à établir un modèle conduisant à interroger la notion même de problème au Moyen Âge. Aujourd'hui, nous voudrions reprendre l'étude des séries de problèmes sous deux angles différents. Le premier consiste à poursuivre l'analyse des transferts et des héritages à l'aide d'un nouveau corpus, celui des recueils de problèmes que l'on dit universitaires, le second à mener, à la suite d'autres, une analyse plus poussée des textes au travers d'une mise en évidence de leur structure grammaticale et logique.
\end{abstract}

\begin{abstract}
Seriating and transmitting problems in late medieval arithmetic in vernacular language. This article proposes to revisit our previous study of a set of problems transmitted in the 15th century through a corpus of arithmetical treatises in the vernacular. The aim here is to discuss the method used in that study to account for the transmission of problems from one text to the other in the corpus. In particular, we show that the computational method we followed led us to establish a model, which implied questioning the very notion of "problem" in the Middle Ages. Now we would like to revisit the study of medieval series of problems through two new approaches. The first consists in continuing the analysis of the transmission processes on a new corpus, namely the corpus of the so-called "university" problems; the second in going deeper into the analysis of texts through the examination of their logical and grammatical structure.
\end{abstract}

\section{Introduction}

Il s'agit dans ces quelques pages de présenter une expérience à présent ancienne, puisqu'elle date de 2007, portant sur l'analyse d'un corpus de manuscrits produits au $\mathrm{Xv}^{\mathrm{e}}$ siècle et témoignant d'un enseignement destiné aux marchands. Ce groupement de documents présente des caractéristiques qui en font un matériau historique de choix. Sur un plan thématique tous les manuscrits partagent un ensemble

\footnotetext{
a e-mail : stephane.lamasse@univ-paris1.fr
}

This is an Open Access article distributed under the terms of the Creative Commons Attribution License 4.0, which permits unrestricted use, distribution, and reproduction in any medium, provided the original work is properly cited. 
de proximités textuelles ou formelles qui renvoient un sentiment d'unité assez fort dès la première lecture $^{1}$. Diachronique, cet ensemble couvre le dernier siècle du Moyen Âge et présente un caractère d'évolution qui peut permettre aussi d'entrer dans une étude de la durée. Enfin, il catalyse des héritages pratiques et universitaires existant depuis le début du XIII ${ }^{\mathrm{e}}$ siècle au moins, et qui rassemblés dans ces textes produisent un sentiment de synthèse et de densité qui a marqué les historiens et l'historiographie.

Dans ce corpus de textes, nous avons pris le «problème ${ }^{2}$ » et sa mise en série comme l'unité élémentaire de comparaison. Pour l'instant, on utilise une définition large du terme " problème », en regroupant ceux qui utilisent des scénarisations ayant pour finalité de pratiquer un algorithme (les pédagogues d'aujourd'hui utiliseraient peut-être le terme d'exercice) et ceux qui mettent en scène une situation supposant de bâtir un raisonnement plus ou moins complexe pour résoudre l'énoncé.

Après un rappel détaillé sur les caractères de cet ensemble documentaire lui-même et sur les démarches empruntées par les études antérieures, nous proposons un retour réflexif sur une expérience de modélisation des problèmes mathématiques contenus dans ces arithmétiques dites «pratiques » écrites en français et en occitan au $\mathrm{XV}^{\mathrm{e}}$ siècle.

\section{Le corpus des arithmétiques "pratiques »}

Le corpus d'arithmétiques pratiques présenté par Guy Beaujouan et augmenté par Jean Cassinet a été produit dans le courant du $\mathrm{Xv}^{\mathrm{e}}$ siècle, entre le premier tiers $\mathrm{du} \mathrm{Xv}^{\mathrm{e}}$ siècle et le début $\mathrm{du} \mathrm{XVI}^{\mathrm{e}}$ siècle. Il se compose d'environ 14 traités possédant tous des caractères assez pratiques, se manifestant notamment par des séries de problèmes mathématiques orientés vers le monde marchand (troc, règle de compagnie, conversion, calcul de prix). Ils mettent au centre de leur démarche des règles d'arithmétique présentées de façon progressive, avec comme point d'orgue les usages de la règle de trois et un ensemble d'autres règles qui peuvent lui être liées. Il ne s'agit pourtant pas de manuels de pratiques marchandes, mais plutôt de textes attestant d'une volonté de présenter une arithmétique appliquée au monde marchand.

Guy Beaujouan faisait remarquer que les ouvrages produits dans le Nord de la France (autour du bassin parisien) avaient une structure textuelle plus proche du modèle de l'algorisme de Sacrobosco ${ }^{3}$. De la même façon, l'influence des recueils de problèmes circulant dans le monde universitaire s'y trouve plus marquée ${ }^{4}$.

Cette empreinte universitaire distingue ces ouvrages des arithmétiques en langue vulgaire qui manifestent un effort de mise en ordre. Dans ce second type de textes, en effet, les problèmes sont regroupés le plus souvent autour de règles dont la succession structure les textes. Cette forme d'ouvrages existe depuis bien longtemps dans le monde italien, dès le XIII ${ }^{\mathrm{e}}$ siècle. Le Liber abaci de Léonard de Pise en est un très bon exemple.

Pourtant dans ces textes le terme « problème » n'est pas utilisé par les auteurs pour désigner une question qui peut être résolue à partir d'éléments donnés dans l'énoncé. On leur préfère les termes « raison» ou « question ». Si le second peut s'entendre comme le début d'un nouveau problème, « raison » est lui plus polysémique, car il désigne aussi fréquemment le calcul. On a pu observer que

\footnotetext{
${ }^{1}$ On peut nuancer cet aspect dans la mesure où dans ce groupement coexistent plusieurs langues, comme l'a relevé (Beaujouan 1998). Voir aussi (Cassinet 1993).

2 En ce qui concerne la notion de série, voir $§$ suivant.

3 Voir (Beaujouan 1954). Jean de Sacrobosco passe pour avoir été l'auteur d'un ensemble de textes utilisés dans le cadre de

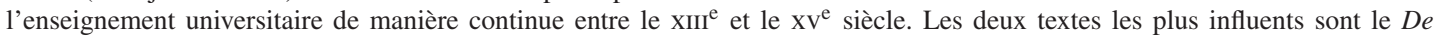
sphera, et l'Algorismus vulgaris. Le succès de l'œuvre de Sacrobosco fut tel que le mot algorismus en vint à nommer la science à laquelle il introduisait dans son traité.

${ }^{4}$ Nous reviendrons sur ce point plus loin. Il s'agit d'une suite de problèmes mathématiques dont l'ordonnancement n'est pas explicite, mais qui sont fréquemment recopiés. Plusieurs d'entre eux ont fait l'objet de traductions à partir du latin vers une langue vernaculaire, comme celui conservé dans le manuscrit 399 de la bibliothèque de Tours, daté du Xv ${ }^{\mathrm{e}}$ siècle. Pour l'instant on ne connaît pas de traduction entre langues vernaculaires.
} 
l'usage de chacun de ces termes était assez clivant dans le corpus. Toutefois, d'autres vocables sont utilisés pour désigner des ensembles de problèmes eux-mêmes, comme « enigmes » (BNF latin 7352) ou « esbatement » (Cesena S-XXVI-6, fol. 113r); il arrive aussi qu'un problème se confonde avec une règle comme la « regle du Lymachon ${ }^{5}$ ». Plusieurs niveaux d'approches des problèmes s'imbriquent donc dans ces traités.

La présence des problèmes, leur mise en série appellent plusieurs remarques :

a) Comment délimiter une série ? La plupart du temps, dans les arithmétiques destinées aux marchands, c'est sous une règle que se déclinent la ou les séries. Ensuite, la décomposition en unités élémentaires constituant la série n'est pas toujours aisée, de sorte qu'un texte peut n'être qu'une déclinaison du problème précédent et lui donner suite. Il est difficile dans ce cas d'arbitrer si un nouveau problème commence, ou bien si cette variante lui appartient. Dans cette première partie de mon travail de modélisation, j'ai fait le choix de séparer les problèmes en fonction de deux paramètres : la présence d'un énoncé et la mise en évidence dans le texte d'un nouveau paragraphe. Cette délimitation s'est avérée insatisfaisante.

b) Comprendre la structure de ces séries est un moyen d'entrer dans le texte. Un problème peut exister dans sa singularité, il peut être usuel - voire participer à une forme de « culture » du problème comme la « regle du lymachon » (BNF 1339, fol. 60) et être un élément dans une suite ordonnée constituant un texte, ce qui est une première forme de mise en série. Il peut aussi se trouver mobilisé, avec d'autres, dans des séquences constituées que l'on retrouve dans d'autres ouvrages. Il faut donc interroger la série dans le sens qu'elle peut prendre à l'intérieur d'un texte, mais aussi dans l'histoire des segments qui la composent. Cette approche permet d'aborder la circulation de savoir à la fois sur un plan diachronique et synchronique. En ce qui concerne le premier point, on peut invoquer l'exemple du manuscrit 2404 de la bibliothèque Riccardiana de Florence, datant de la fin du XIII ${ }^{\mathrm{e}}$ siècle et édité par (Gino 1989). Ce dernier propose des mises en séries quasi-thématiques, où les ensembles de problèmes reçoivent des intitulés comme « dix-huitième chapitre, portant sur l'homme qui trouve une bourse ${ }^{6} »$.

Cependant, tous les problèmes mobilisés dans l'écriture ne trouvent pas forcément de solution dans le cadre de règles clairement identifiées, comme c'est le cas pour la règle dite d'apposition remotion (Benoit et Lamassé 2003), dont les auteurs ne donnent pas tous de définition et qu'ils n'utilisent pas de la même façon. Il arrive ainsi qu'il n’y ait pas de règle permettant de regrouper des problèmes, mais tout un ensemble de procédures dans des parties parfois nommées « esbatement ».

c) Dans le cas d'une règle, l'étude des séries de problèmes peut renseigner autant sur la façon dont on veut donner à comprendre cette règle que sur les logiques de composition de l'ouvrage dans sa globalité. C'est en tout cas ce que l'on peut observer en comparant le premier traité du manuscrit S-XXVI-6 de Cesena avec le Triparty en la science des nombres de Nicolas Chuquet.

d) Enfin, la circulation et la production des problèmes est relativement importante pour le Moyen Âge et encore assez peu étudiée. Il existe pour le bas Moyen Âge occidental d'autres corpus que celui des arithmétiques marchandes, constituant un fonds de problèmes assez nombreux, parce que la quantité de manuscrits est importante. Il s'agit de celui des recueils universitaires. Les problèmes qu'ils renferment ne sont pas très variés. La suite de ces problèmes semble connaître peu de mouvement d'après les éditions existantes. Ces séries ont été copiées dans de multiples langues (latin, italien, français, allemand, espagnol). C'est une documentation probablement assez importante. Ce « fonds documentaire » est mobilisé par certains des manuscrits d'arithmétiques en langue vulgaire.

\footnotetext{
5 «Limachon » a le sens de « limaçon ». Nous n'épuisons pas ici les dénominations des problèmes, il s'agit simplement d'en montrer la variété. Le dernier phrasème me semble renvoyer à une forme de désignation du problème discutée par (Genthilhomme 1995).

6 «L'octavodecimo chapitolo è de huomene che trovaro borse» [fol. 74r], p. 81 dans Gino Arrighi, op. cit. Dans ce texte tous les problèmes semblent être organisés de la même façon.
} 
Tous ces points rendent l'étude des circulations possible et souhaitable. Les arithmétiques en langue vulgaire française ou provençale, bien que très structurées, laissent transparaître des héritages différents et la rémanence de textes qui continuent à être produits dans d'autres cadres que le monde marchand. On rejoint donc, au travers de ce corpus d'une dizaine de manuscrits, des problématiques plus larges portant sur l'étude des problèmes mathématiques, dont un parcours historiographique peut permettre de positionner et de donner à comprendre la perspective que nous avons retenue.

\section{Quelques éléments d'historiographie}

Les problèmes représentent des moments clairement identifiables d'une activité mathématique. Il est probable que c'est une des raisons qui expliquent l'attachement des historiens. Et cela est particulièrement vrai pour ceux qui se sont intéressés au Moyen Âge, dont la documentation relativement importante augmente entre le $\mathrm{v}^{\mathrm{e}}$ et le $\mathrm{XVI}^{\mathrm{e}}$ siècle. Si cet accroissement est perceptible, les contextes d'apparition des problèmes dans les manuscrits évoluent et semblent témoigner de tendances plus profondes, susceptibles de sourdre dans l'étude attentive non seulement des problèmes pour eux-mêmes, mais aussi des séries que leurs enchaînements textuels constituent.

\subsection{Jusqu'au XIX ${ }^{\mathrm{e}}$ siècle : regrouper et transmettre}

Écrire l'histoire de ces problèmes est aussi une façon privilégiée de construire une histoire des mathématiques. Une histoire peut-être moins attachée aux grands savants, mais qui ne les met pas de côté a priori. Au XIX ${ }^{\mathrm{e}}$ siècle, le goût pour la collection s'est accompagné d'un souci de recherche sur l'histoire des mathématiques. Ainsi Édouard Lucas complète-t-il le livre de Claude-Gaspard Bachet, édité pour la première fois en 1612, en introduisant une biographie de l'auteur et en essayant de mettre en relation certains problèmes. C'est ainsi que se constituent les bribes d'une généalogie qui connaît un certain succès éditorial. Les Mathematical Recreations and Essays de Walter William Rouse Ball, qui classent les problèmes en fonction des périodes et des domaines mathématiques, ont été traduits en plusieurs langues et souvent réédités. Ces éditions, nourries par les découvertes de leur temps, écrivent déjà une histoire des mathématiques par le truchement de la généalogie des problèmes qui émergent dans les dernières éditions. Aussi n'est-ce peut-être pas un hasard si les premiers travaux prenant les problèmes comme objet d'histoire sont presque contemporains des précédents recueils cités, puisqu'ils datent de la fin du XIX ${ }^{\mathrm{e}}$ siècle. Il s'agissait alors d'inventorier les problèmes en les classant dans des catégories et ainsi de repérer des héritages, des emprunts. Cette histoire a intéressé des Allemands, comme Maximilian Curtze, qui en 1895 amorce une démarche historique en proposant l'édition d'un recueil de problèmes du Moyen Âge et, à la fin de son article, un petit commentaire mathématique des problèmes. Dans ces notes, il essaie parfois de donner l'origine de certains problèmes. Mais c'est en 1902 que Johannes Tropfke dans son ouvrage Geschichte des Elementar-Mathematik réalise la première typologie des problèmes. Cet ouvrage, sans cesse réédité, fut enrichi constamment, pour devenir aujourd'hui le principal et le plus précis des inventaires que l'on possède. À sa suite, en 1917, David Eugene Smith propose de nombreuses pistes dans « On the origin of certain typical problems ». Il s'agissait de reconstruire au travers des problèmes une histoire de la civilisation. Un peu plus tard, en 1925, il consacre une partie de son History of Mathematics aux mêmes problèmes, puis dirige la thèse de Vera Sanford, The History and Significance of Certain Standard Problems in Algebra, publiée en 1927. C'est la première fois qu'une étude considère les problèmes mathématiques comme objet. L'auteure s'interroge dans ses deux premières parties non seulement sur les raisons de l'utilisation d'exercices verbalisés, mais aussi sur ce que doit être un bon problème, et livre un sondage sur l'intérêt des écoliers pour ceux-ci. Elle entend réfléchir sur le statut du problème dans l'enseignement des mathématiques à son époque. Vera Sanford se fixe un troisième objectif : trouver les facteurs permettant d'expliquer qu'un problème se perpétue ou soit abandonné. L'étude est assez large puisque sont considérés comme 
algébriques les problèmes que l'on pourrait résoudre aujourd'hui par l'algèbre. Ce travail n'est pas une synthèse exhaustive susceptible de fournir des renseignements précis sur la transmission des problèmes, mais un aperçu fait à partir de manuscrits édités ou imprimés. L'intérêt historique de l'ouvrage est évident, puisque c'est le premier essayant de dégager tout ce qu'un exercice peut donner comme informations à l'histoire des mathématiques. Vera Sanford se dégage donc d'une simple perspective généalogique.

\subsection{L'élargissement des problématiques}

La généalogie a connu un certain succès auprès des historiens, non pas tant pour dégager des origines que pour percevoir une circulation des savoirs mathématiques sur de grands espaces. Cette tendance connaît, depuis quelques décennies, un renouveau important. En 1983, Kurt Vogel propose de retracer l'histoire des problèmes d'un livre de calcul byzantin du XIV ${ }^{\mathrm{e}}$ siècle, à travers un arbre chronologique et culturel permettant d'observer les héritages sur une échelle de temps très longue $\left(\mathrm{v}^{\mathrm{e}}-\mathrm{Xv}^{\mathrm{e}}\right.$ siècle $\left.^{7}\right)$. $\mathrm{C}^{\prime}$ est dans la même perspective d'héritages, de traditions, que s'inscrit l'édition, par Menso Folkerts, des Propositiones d'Alcuin en 1978 (Folkerts 1978). Par cette édition l'auteur entend relancer l'histoire des problèmes en proposant une des sources les plus importantes du Moyen Âge. Dès 1971 il avait réalisé une étude des cautele, enigmata, subtilitates anonymes des manuscrits latins extraits de la Bodleian Library, du British Museum, de la bibliothèque de Berlin, de celles de Munich, Vienne et Erfurt. La difficulté inhérente à cette histoire des transmissions de problèmes tient à la quantité de travail que nécessiterait une étude exhaustive. Ainsi, une étude globale doit attendre, comme le rappelle Warren Van Egmond (Van Egmond 1996, p. 379-428), que des études plus ponctuelles, adoptant une démarche commune, aient été réalisées.

Parallèlement un autre type de démarche est développé dans les années 1950-1960. En 1954, Guy Beaujouan propose un nouveau regard sur les problèmes mathématiques des manuscrits latins, puisqu'il envisage, à travers une étude d'ensemble (XIII $-\mathrm{XIV}^{\mathrm{e}}$ siècle), la pratique des mathématiques chez les étudiants de l'Université. Il ne s'agit plus seulement de comprendre des généalogies, mais de s'intéresser à la façon dont les recueils de problèmes pouvaient être lus par le public des étudiants de cette période en complémentarité avec d'autres textes didactiques que sont l'Algorismus de Sacrobosco et le Carmen de algorismo d'Alexandre de Villedieu.

Plus tard, en 1962, Ernest Coumet écrit un ensemble d'articles sur « le problème des partis avant Pascal », où il analyse l'évolution de la formulation de l'énoncé de ce problème et certaines réponses apportées par différents mathématiciens. Il recherche aussi les problèmes qui sont à l'origine de celui-ci pour mieux comprendre avec quelles références pensaient les hommes dont il retrace le raisonnement. Dans cette perspective, l'histoire des problèmes permet de retracer le cadre mental des mathématiciens.

En 1983, Kurt Vogel donne la première étude comparative portant sur un seul problème ayant pour finalité le calcul de la hauteur d'une montagne. Cette démarche est reprise en 1997 par Harald Gropp à travers l'histoire d'un exercice apparaissant en Occident dans les Propositiones ad acuendos juvenes d'Alcuin ${ }^{8}$. Cet article présente plusieurs intérêts. L'un d'entre eux est de montrer que cet exercice est commun à plusieurs cultures (africaine, nord-africaine, océanienne). Ensuite, il en analyse le rôle fondateur pour la théorie des graphes, ce qui le conduit à mener une réflexion épistémologique sur la place des graphes dans les mathématiques. En 1999, Maryvonne Charrier-Spiesser a soutenu une thèse où une étude précise de quatre problèmes du Compendy de la pratique des nombres, replacés dans leur contexte mathématique grâce à la comparaison avec d'autres textes ayant des problèmes voisins, a permis de montrer toute l'originalité de cet auteur et d'amorcer une véritable réflexion sur

\footnotetext{
${ }^{7}$ Effort de catégorisation des problèmes à la fin de son édition : (Vogel 1954).

${ }^{8}$ Il s'agit de celui du loup, de la chèvre et du chou qu'un homme doit faire passer par une rivière, sans que le loup soit avec la chèvre, ni la chèvre avec le chou (Gropp 1998, 31-41.)
} 
le corpus de problèmes dont dispose le Moyen Âge (Spiesser 2001). En 2004, Marie-Hélène Labarthe a proposé une étude comparée très fouillée de trois arithmétiques du $\mathrm{XVI}^{\mathrm{e}}$ siècle. Elle a eu recours à une formalisation mathématique méticuleuse qui lui a permis une comparaison pointue de la diversité des méthodes algorithmiques qu'elle a relevées. Cette démarche a autorisé la mise en évidence d'une forme de hiérarchisation des problèmes sous forme de ramification plus ou moins importante. Elle permet d'entrevoir les raisons expliquant les choix des problèmes chez certains auteurs, et notamment chez l'auteur de la Suma de Santcliment. Elle montre que l'usage d'algorithmes spécifiques conditionne la richesse de la diversité des problèmes retenus. Elle met en place des conditions de comparaison des problèmes qui pourraient être réutilisées.

Ces différentes approches sont cumulatives et il est bien évident que les problèmes peuvent et doivent être historicisés. Cette perspective, pour simple qu'elle puisse apparaître, permet d'introduire plus clairement la notion de contexte ${ }^{9}$. Ce qui induit l'idée simple qu'un problème peut difficilement faire l'objet de la même opération de lecture hier et aujourd'hui, mais aussi que sa mobilisation, une ou plusieurs fois, à l'intérieur d'une même œuvre, ou de deux œuvres différentes, constituent autant d'événements distincts. Il faut dès lors comprendre les logiques de regroupement des problèmes. L'analyse des problèmes et leur mise en série est un point d'intersection entre une histoire de la circulation des savoirs, la compréhension des logiques de construction d'un ouvrage et les mathématiques mises en œuvre. C'est par conséquent un champ d'étude à part entière.

Sur un plan méthodologique, si l'on veut se donner une chance d'appliquer les différentes façons d'étudier les problèmes dans les œuvres, il faut pouvoir les mobiliser rapidement pour les comparer sur des critères communs. C'est pourquoi la construction d'une base de données pouvait paraître intéressante. En déstructurant les problèmes et les ouvrages pour fabriquer un catalogue, elle conduisait à centrer la réflexion sur le « problème » et à interroger globalement et finement les données accumulées.

\section{Intérêts et résultats de la mise en place d'une base de données}

\subsection{Présentation générale}

La Figure 1 présente un modèle conceptuel de la base de données, dont l'objectif est de permettre une comparaison raisonnée de tous les problèmes du corpus. Au delà de cette attente, la constitution de la base de données m'a poussé à rendre explicites un certain nombre de choix et à faire apparaître la façon dont j'ai pu me représenter le problème et son étude dans le cadre de ce travail. Le graphique joint facilite d'ailleurs la lecture et le partage de cette modélisation. Cette dernière a été produite en deux temps, assez caractéristiques du travail d'historien, qui fonctionne beaucoup sous forme d'agrégation ${ }^{10}$. Le premier a été un parcours de la documentation pour construire le modèle et le second l'adaptation continue de ce modèle aux nouvelles situations qui ne manquent pas de se présenter chaque fois que l'on ouvre un nouveau manuscrit.

Comme on peut l'observer sur le schéma, le modèle tient compte de trois échelles, celle des ouvrages, une autre plus proche de la mise en série de problèmes dans les manuscrits étudiés et enfin une dernière centrée sur le problème dans sa singularité. La part de description des manuscrits inclut des éléments très simples dont la finalité est à la fois de permettre une interopérabilité avec d'autres bases de données (notamment Jordanus ${ }^{11}$ ) et de mettre en relation le traité considéré avec l'ensemble de ceux d'un manuscrit. On considère, en effet, que chaque manuscrit peut contenir plusieurs traités. La description des traités était assez rapide, car chaque traité correspondait le plus souvent à un

\footnotetext{
9 Voir à ce propos l'introduction de (Bernard et Proust 2014).

${ }^{10}$ Sur l'intérêt de ce travail de modélisation dans une perspective réflexive, voir (Genet 1986 ; McCarty 2014), je remercie Camille Salinesie pour sa relecture.

11 http://archimedes.mpiwg-berlin.mpg.de/data.htm (consulté le 29.10.2015). Il s'agit des tables de localisations : pays, villes, bibliothèques, manuscrits.
} 


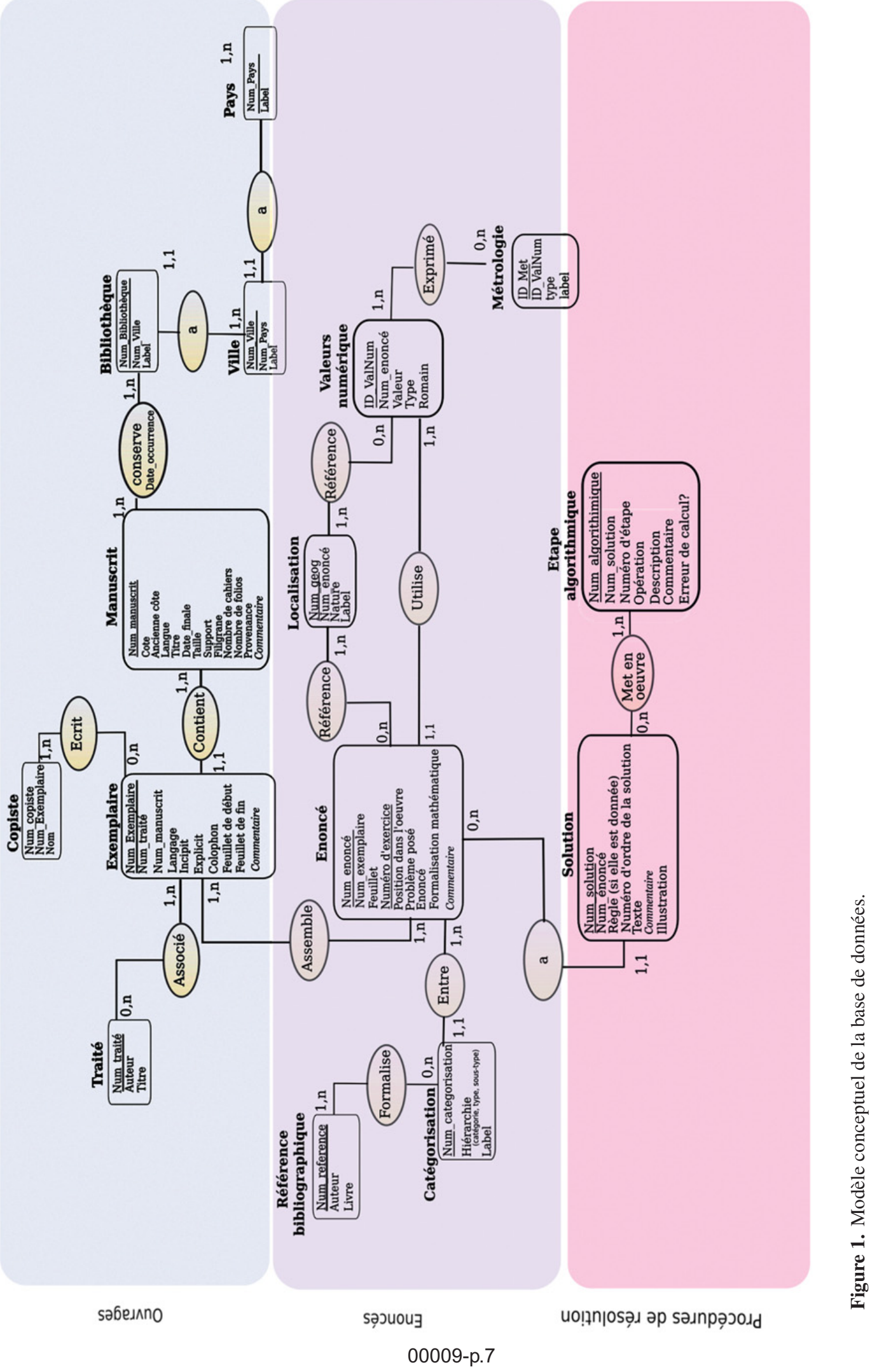


unique manuscrit. Il était important, au demeurant, d'envisager qu'un manuscrit puisse contenir plusieurs traités et qu'un traité était potentiellement, même sous forme de fragments ${ }^{12}$, dans plusieurs manuscrits. Toutefois, cette situation étant possible elle a été introduite dans la base de données.

\subsection{Caractérisation des énoncés}

La suite des énoncés est enregistrée en tenant compte de leur position dans l'ouvrage (feuillet, " position dans l'œuvre ${ }^{13} \gg$ ), ainsi que de leur position relativement aux autres problèmes (« numéro d'exercice »), qui est un indicateur de sérialité. L'énoncé a été reproduit, accompagné lorsque cela était possible d'une formalisation mathématique contemporaine et donc anachronique. Un certain nombre d'attributs concernant les énoncés ont été enregistrés. Parmi ces attributs, il y a le texte évidemment, car je souhaitais dégager des structures dans les méthodes d'énonciation. Il y a, en effet, plusieurs éléments de langage dans ces problèmes. Le premier est factuel, il s'agit d'éléments faisant l'objet de transformations d'un traité à l'autre et qui témoignent fréquemment d'une adaptation du texte. Mais l'usage de la langue peut être analysé d'une façon plus grammaticale, en rapport avec l'organisation même de l'énonciation ${ }^{14}$. Dans tous les cas, on a enregistré le fait que le problème est introduit par des éléments de continuité («Item », « Item plus », « plus », etc.), ou bien encore que le problème est proposé sous forme affirmative ou interrogative.

Le présupposé étant de retrouver les problèmes identiques, ou voisins, il était nécessaire d'introduire tout de même un certain nombre d'éléments de description autorisant ces rapprochements. Le problème est conçu comme englobant l'énonciation, la ou les solutions, les preuves ainsi que les mises en œuvre graphiques. Pour entrer dans la base de données, il faut avoir un énoncé, il n'est pas possible d'entrer avec la seule solution. Cette approche m'a posé une difficulté dans la mesure où, comme on l'a vu plus haut, il est parfois difficile de délimiter un problème. Des transformations d'énoncés n'étant dans ce cadre que des variantes, j'ai introduit dans la table un attribut «variante du précédent ». Cette approche déplaçait la difficulté sans la résoudre vraiment, en augmentant les niveaux de description. La structure des textes que nous avons pu lire a tendance à isoler chaque transformation d'énoncé par un retour à la ligne, c'est pourquoi nous les avons enregistrées à la suite.

Parmi les éléments caractérisant un énoncé, on a conservé ceux relatifs aux systèmes métrologiques employés, les références à des espaces géographiques, ainsi que les valeurs numériques. J'avais d'abord imaginé une description plus précise de chaque problème, en enregistrant toutes les données factuelles : nombre et nature des individus, objets, animaux présents dans les énoncés. L'alourdissement de la saisie devenant conséquent, j'ai privilégié la rapidité de saisie des énoncés.

Enfin, si un énoncé était présent dans un ouvrage antérieur ou postérieur, les références étaient introduites dans le gisement de données.

\subsection{La prégnance de l'historiographie dans le modèle}

La catégorisation des problèmes mathématiques, pour importante qu'elle soit, introduit directement l'historiographie dans la base de données. Typer un problème, c'est d'abord prendre position par rapport à la façon dont les historiens précédents avaient construit des catégories ${ }^{15}$. Or je souhaitais que la base de données soit sensible, au moins dans un premier temps, le moins possible, au poids des

\footnotetext{
12 Il existe des fragments de traités dans plusieurs manuscrits.

13 Si une structure a été donnée au texte, elle est indiquée ici. Cela permet de recoller avec l'ensemble des textes transcrits par ailleurs.

${ }^{14}$ C'est aussi pour cette raison que l'ensemble des textes a fait l'objet d'une transcription assez précise incluant tous les éléments graphiques du texte.

15 Comme nous l'avons vu dans la partie historiographie (sous-partie 3), il y en avait deux grandes constructions typologiques, celle du manuel de Tropfke, largement revue par les historiens allemands postérieurs, offrant l'avantage non négligeable de pouvoir
} 
historiens précédents. Il s'agissait de le reculer et non pas de le proscrire. Aussi était-il préférable de fabriquer une table particulière dans la mesure où chaque problème peut être catégorisé de plusieurs façons et que chacune d'elles peut qualifier plusieurs problèmes. Les énoncés ne sont pas dépendants d'une typologie, mais appartiennent à plusieurs méthodes de catégorisation.

Dans cette phase de la modélisation des données, nous avons été sensibles à l'idée de concilier les problèmes transcrits et la perception que l'historiographie pouvait en avoir. L'avantage était d'accroître nettement le catalogue des problèmes en utilisant ceux référencés par les différents historiens, et de favoriser les comparaisons, sans tenir compte de contraintes linguistiques. Ce modèle permettait de reproduire la structure de l'œuvre mais laissait de côté les règles lorsqu'elles existaient : le texte de celles-ci a donc fait l'objet d'une reproduction dans la transcription exhaustive des textes.

\subsection{Les solutions}

Chaque énoncé peut avoir une ou plusieurs solutions dont on conserve le texte, le numéro d'ordre, les commentaires s'ils existent, la règle utilisée dans cette solution. Dans le schéma suivant un même énoncé peut donner lieu à plusieurs solutions. Chaque solution met en œuvre plusieurs étapes, dont l'ensemble restitue un algorithme. Mais comment décomposer ces étapes ? Là encore, il n'est pas si simple de « décider » d'une étape. Évidemment le texte, souvent prescriptif, nous guide et on peut dégager assez bien une suite d'opérations à réaliser. Mais très vite les textes ont tendance à abréger, à donner des locutions qui renvoient à des procédures acquises - on ne sait pas toujours lesquelles... Aussi, nous avons opté pour une solution simple, fondée sur l'ensemble des étapes décrites par le texte, en prêtant une attention fine aux connections logiques et aux implicites. Lorsqu'une locution conseille d'opérer comme on le fait avec une autre règle, comme la règle de trois (" tu fais une règle de trois »), on n'a pas décomposé les étapes mais enregistré cela comme une étape. De même pour la division ou la multiplication. Ce qui signifie que si l'on cherche à restituer les calculs, il faut tenir compte des sous-étapes. Et très souvent ces « sous-étapes » sont les lieux d'une interprétation de l'historien : c'est pourquoi nous avons adjoint l'attribut « commentaire ».

\subsection{Les dispositifs graphiques}

La table « dispositif graphique » vise à intégrer toutes les représentations (dessins, opérations) introduites dans le texte. Si l'existence d'une telle entité peut sembler évidente a priori, la distinction entre le texte et l'image ne l'est pas. Cette simplification facilitait l'étude à court terme, en permettant l'inventaire de ce qui est posé, mais occultait une part relativement importante des informations. Une des questions que je me suis posées consistait à savoir si l'on peut considérer des éléments de texte comme des dispositifs graphiques. Ainsi, pour la règle de trois, on trouve différentes procédures de mise en forme spatiale de l'énoncé (voir infra Sect. 6.2). C'est un des objectifs de ces textes que de faire acquérir des techniques graphiques de résolution de problèmes.

\section{Quelques résultats}

\subsection{La cohérence du corpus}

L'ensemble des problèmes introduits dans la base de données permettait de construire un indice de liaison entre les manuscrits (Fig. 2). Cet indice était fondé sur les énoncés identiques. Sans surprise, deux

comparer avec des ensembles beaucoup plus vastes de problèmes, donc un intérêt documentaire. L'autre a été émise par Warren Van Egmond mais se trouve assez convergente. 


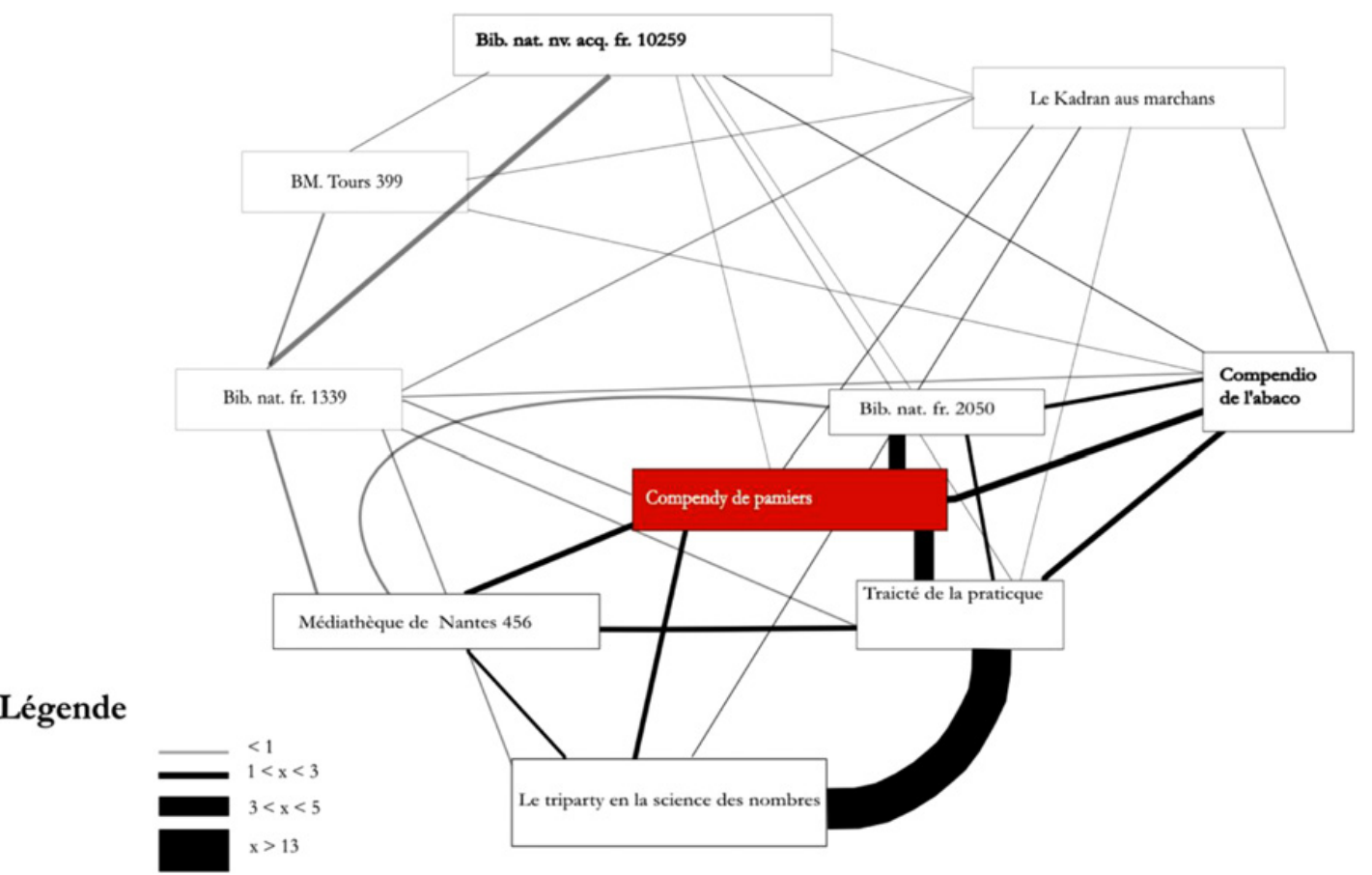

Figure 2. Les relations entre les manuscrits au travers de la circulation de problèmes strictement identiques.

constatations émergent. La première confirme l'existence d'une tradition centrée sur deux manuscrits ${ }^{16}$ qui apparaissent très structurants pour ce corpus, pour des raisons différentes ; l'autre, qu'il existe un ensemble de problèmes qui constituent une forme de culture commune à tous les ouvrages. Certains problèmes doivent être présents, ils sont peut-être même attendus - peut-être sont-ils des passages obligés, non seulement parce qu'ils seraient l'archétype d'une règle mathématique, mais aussi parce qu'ils auraient une vocation culturelle dans un espace sociale plus large.

\subsection{La comparaison des manuscrits deux à deux}

On peut ensuite se proposer de comparer systématiquement deux à deux les ouvrages, la Figure 3 (annexes) en donne un exemple. Elle présente sur deux colonnes la suite des problèmes dans chacun des manuscrits, dans leur ordre d'écriture. Les grandes segmentations du texte sont rappelées en gras. Lorsque deux énoncés sont identiques, le folio et le numéro du problème apparaissent vers le centre côte à côte ; dans le cas contraire, ils sont écartés soit vers la gauche du graphique, soit vers la droite. Les ajouts deviennent clairement visibles et les reclassements sont indiqués par des traits reliant les numéros des ouvrages concernés entre eux.

Dès lors, ce qui devient important, ce n'est pas seulement la quantité de problèmes identiques, mais bien le nombre de séries identiques. Ce qui ne va pas sans difficultés, car il faut envisager les différences de taille des séries ainsi que leur continuité, car il peut arriver qu'elles soient discontinues, voir interrompues.

\footnotetext{
16 Il s'agit du BNF nv. ac. 4140, produit à Pamiers, et de l'ouvrage copié par Mathieu Préhoude, Cesena S-XXVI-6. Ces manuscrits ont fait l'objet de nombreuses présentations, comme celles, pour le premier, de (Sesiano 1984) et, pour le second, de (Fery-Hue et Spiesser 2007).
} 


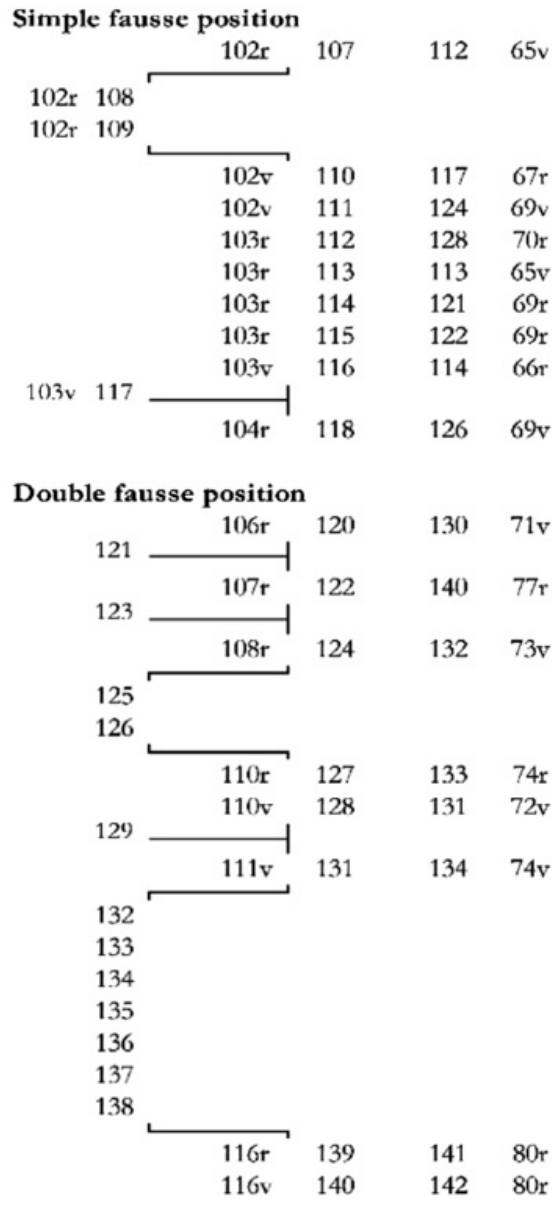

Figure 3. Table de comparaison des textes deux à deux.

\subsection{Le mouvement des séries}

Ces résultats ne font que marquer le début du travail : il faut essayer de comprendre ce que signifient exactement ces séries et leurs réarrangements à l'échelle des traités eux-mêmes et entre des traités différents. De fait, il n'y a pas de raison a priori pour qu'il existe des séries complètement figées dans le temps. Au contraire, le mouvement des séries peut témoigner de réorganisations de savoirs témoignant des finalités différentes des œuvres écrites. L'exemple le plus évident que nous ayons de cette situation concerne les relations des textes de Mathieu Préhoude et de Nicolas Chuquet (Lamassé 2007) : le second reprend des séries de problèmes entières du premier pour les réordonner dans sa perspective propre.

C'est un des aspects que j'aimerais développer dans le cadre d'une source un peu particulière, celle des « recueils » de problèmes qui ont circulé dans le milieu universitaire à partir du XIII ${ }^{\mathrm{e}}$ siècle et dont l'analyse du contenu n'a pas fait l'objet d'une telle approche. Il s'agirait de reprendre le sondage réalisé par Menso Folkerts en 1971 en essayant de voir s'il existe une ou plusieurs traditions de ce type d'ouvrages et si ceux-ci connaissent des transformations significatives dans le temps. On peut se demander si les changements dans les séries n'est pas un indicateur de la circulation et de la diffusion d'autres textes. 


\subsection{Entrer dans l'écriture des textes}

Comprendre comment les séries s'organisent, sont copiées ou construites est une façon d'entrer dans l'écriture du texte ${ }^{17}$. Le manuscrit de Nicolas Chuquet donne le sentiment d'agrégation, de collection ordonnée de problèmes, et il n'est pas rare dans la partie sur « les premiers » de trouver des ajouts en marge. Jean Certain, l'auteur du Kadran aus marchans, propose une progression pour chaque règle allant de l'exemple applicatif à la règle jusqu' au problème limite.

\section{5 Étendre le corpus ?}

La construction d'un catalogue permet des comparaisons plus complexes à plusieurs niveaux. La difficulté essentielle est le passage à l'échelle, c'est-à-dire l'augmentation du nombre des problèmes enregistrés dans la base de données. Si la série de problèmes mathématiques dans le cadre des œuvres devient l'objet de la recherche, le corpus des traités considérés est continuellement en expansion. Ces traités ouvrent sur un ensemble beaucoup plus vaste, qui s'étend dans l'espace et dans le temps. Il faut entendre par là l'Italie à partir du XIII ${ }^{\mathrm{e}}$ siècle, l'Espagne à partir de la fin du XIV ${ }^{\mathrm{e}}$ siècle, le monde germanique et anglo-saxon. À cela, il faut ajouter les ouvrages en latin.

En effet, le corpus peut s'étendre à plusieurs langues - textes latins, italiens, anglais, allemands qui deviennent comparables ${ }^{18}$ - ou bien à d'autres domaines, comme le métier de changeur ou les problèmes plus « ludiques » que l'on trouve dans les devinettes françaises éditées par Bruno Roy ${ }^{19}$. Les usages de ces problèmes sont en effet très différents et leur contexte d'apparition n'est pas à négliger. La comparaison de textes entre différentes langues entraîne des difficultés évidentes dont nous avons soulevé quelques aspects.

Un tel travail déborde de loin celui qu'un chercheur solitaire peut fournir. C'est la raison pour laquelle Warren Van Egmond proposait plutôt un travail collectif (Van Egmond 1996). Toutefois, pour l'instant, malgré les limites inhérentes au mode d'acquisition de la donnée, j'ai choisi de continuer ce travail seul en restructurant l'information.

\section{Plusieurs modes de lectures : perspectives de recherche}

\subsection{L'étude de la langue et de la syntaxe des problèmes}

Pour fabriquer un espace de comparaison raisonné des problèmes à l'aide d'une base de données, on ne peut se passer d'un élément essentiel, qui échappe partiellement encore à cette approche quantitative : celui de la langue et de sa mise en forme dans le texte, c'est-à-dire non seulement le vocabulaire, mais aussi la syntaxe. Il reste que tout un ensemble de limites demeurent dans le texte enregistré, dépourvu d'un enrichissement fin. En effet, si la base de données présentée ci-dessus peut permettre un certain niveau de comparaison des problèmes, qui nécessite l'arbitrage humain pour décider de la proximité de ceux-ci, elle ne permet pas d'accéder dans sa forme actuelle aux éléments d'énonciation et de résolution. Ces derniers n'ont pas fait l'objet d'une modélisation particulière, parce qu'une telle approche n'est pas entièrement convergente avec le corpus de la base de données, notamment du fait des différences linguistiques. Énoncés et solutions sont présents dans ce gisement de données, puisqu'ils ont fait l'objet d'une transcription.

\footnotetext{
17 Et permet de continuer, sur un autre plan, l'enquête débutée dans (Lamassé 2014).

18 Soulignons qu'un assez grand nombre d'éditions et d'études de ces problèmes sont disponibles.

19 Voir (Roy 1977). Nous avons tenté dans le cadre d'un séminaire d'entrevoir les relations que ces devinettes pouvaient entretenir avec les textes des arithmétiques pratiques et de comprendre la singularité des séries présentes dans le texte : voir http://problemata.hypotheses.org/114.
} 
Ce n'est pas que la langue des arithmétiques en langue vulgaire n'ait pas été étudiée, mais les études se sont concentrées avant tout sur le vocabulaire ${ }^{20}$, à la fois pour en faire l'inventaire et pour affiner la typologie de ces textes pratiques du $\mathrm{Xv}^{\mathrm{e}}$ siècle. En effet, analyser la langue mobilisée dans ces textes permet de focaliser l'attention sur les particularités lexicales des textes considérés et d'apporter des éléments concernant la production de ces textes et les traditions culturelles qu'ils véhiculent.

En ce qui concerne l'évolution de la langue mathématique du Moyen Âge, l'étude la plus générale a été produite par Hervé L'huillier en 1994. Ce dernier souligne les perspectives de recherche ouvertes par cette approche, en s'intéressant aux dynamiques d'évolution sur l'ensemble de la période. Plus récemment, les problématiques se sont orientées vers la construction d'une langue de spécialité pour les mathématiques au Moyen Âge (Frankwalt 2006), ce qui suppose de confronter cette langue avec d'autres domaines de spécialité et d'observer les éléments qui peuvent leur être communs, au moins en apparence.

L'étude fondée sur la langue pousse à délimiter un corpus plus réduit, car les textes étudiés doivent partager une unité de langue, ce qui conduit à ne retenir qu'un ensemble de 12 textes $^{21}$. En revanche, en toute rigueur il faudrait prendre l'intégralité des œuvres, et nous n'avons conservé que les textes les plus orientés vers la pratique commerciale.

Le projet «Série de problèmes » conduit à interroger autrement ce point de vue sur les mots, en se centrant davantage sur la syntaxe et les articulations entre les résolutions et les énoncés proposés, notamment dans le travail de Jeffrey Oaks et Alain Bernard. Il est tentant d'adopter une approche similaire afin d'essayer de comprendre la rhétorique à l'œuvre dans les procédés de résolution.

Dès lors, le retour à une attention plus soutenue à la linéarité oblige à observer des termes qui n'ont pas forcément de sens mathématique mais qui entrent dans l'organisation de la lecture et de la procédure. Il existe, par exemple, des termes-outils permettant d'indiquer une forme de continuité ou de rupture entre des problèmes. La façon de les introduire chez les différents auteurs n'est pas identique. Ainsi le manuscrit BNF 1339, du Nord de la France, introduit généralement les problèmes par « question » et les solutions par « response », mais il n'est pas le seul : le Kadran aux Marchans produit à Marseille en 1485 fait de même. Le texte copié par Mathieu Préhoude a plutôt recours plus à « Item » pour introduire chaque problème, tandis que Nicolas Chuquet utilise fréquemment «plus ». Entre les deux, il y a, me semble-t-il, deux rapports aux problèmes : le premier a tendance à les singulariser, le second à les ancrer dans une série. Dans la première situation, il semble que la question devienne comme un archétype que l'on manipule.

\section{2 Écriture et figuration des procédures}

Un des éléments qui m'ont semblé convergents avec les autres approches présentées dans le cadre du séminaire «Séries de problèmes » concerne la dynamique existant dans ces textes entre l'écriture de la procédure et sa figuration. Le cas de la règle de trois est assez significatif, me semble-t-il22 Cette dernière suppose une formalisation claire du problème afin d'appliquer la procédure de calcul. La difficulté est ici la façon dont on fait apparaître, à partir de l'énoncé, les termes qui vont permettre l'application de la procédure. L'objectif est d'obtenir une formalisation du type « si ...., [que/combien]... ? ». On peut observer cette démarche lorsque l'énoncé ne fait pas apparaître les termes

\footnotetext{
20 À propos de ces différentes approches, je renvoie pour plus de commodité à (Lamassé 2012).

21 Archiloge Sophie, BNF lat. 7352, anonyme (déb. Xve), 4, BNF lat. 7381, anonyme (milieu Xve), BNF fr. 1339, anonyme (v. 1460), BNF fr. 2050, anonyme (v. 1460), Cesena Biblioteca Malestiana S-XXVI, Barthélemy de Romans 1476, Bibl. SainteGeneviève 3143, Jean Adam, 1475, Méd. Nantes 456, anonyme (1480-1490), BNF fr. 1346, Nicolas Chuquet, Le Triparty en la science des nombres, 1484, BNF, Arsenal 2904, Kadran aux marchans, Jean Certain, 1485, Florence Biblioteca Laurentiana, Plut. 29 cod. 43, anonyme (fin XV ${ }^{\mathrm{e}}$ ), BNF nv. acq. fr. 10259, petit cadran, Jean Certain (déb. XVI ${ }^{\mathrm{e}}$ ). On pourra comparer ce corpus aux textes occitans, que l'on sait proches.

22 J'ai essayé de développer ces aspects dans (Lamassé 2012).
} 
immédiatement, comme dans le cas des problèmes « contrefactuels », qui se présentent comme dans cet exemple : «Si 7 valoit 9 quans en fault pour vailloir ou gaigner 17 ? (Hoyrup 2009). Dans certains ouvrages, comme celui de la Biblioteca Malatestiana de Cesena, la formalisation devient pratiquement graphique (Lamassé 2012). Dans les premiers problèmes de la règle de trois, la séquence se déroule comme une progression qui permet de construire cette représentation : « Se $\mathrm{A} / \mathrm{B} / \mathrm{C}$ ». Régulièrement on retrouve ce type d'expression pour exprimer la règle de trois, suivi de « Multiplie et partiz ».

\subsection{Des approches complémentaires}

En définitive, ces deux approches sont complémentaires en ce qu'elles se fondent sur la pratique mathématique et l'écriture des procédures. Elles n'interviennent peut-être pas aux mêmes échelles. La base de données permet de comparer très vite des ensembles de données structurés par le chercheur, en autorisant des manipulations des paramètres de l'énoncé (valeurs numériques, métrologie, etc.). Comme le soulignait Van Egmond, étudier ces aspects est d'autant plus important qu'il s'agit non seulement d'une culture qui dépasse parfois le cercle des mathématiciens, et pas seulement pour les jeux. En outre, ils permettent d'accéder à une culture commune, parfois élémentaire, mais pas toujours. Au Xv siècle, on perçoit mieux ces deux derniers aspects.

Nous n'avons pu tester dans le cadre de ce séminaire une étude fouillée de l'argumentation et le choix que nous avons fait se fondait pour l'instant essentiellement sur des méthodes de lexicographie quantitative. Nous avons eu une approche lexicale et syntaxique, acceptant une lemmatisation forte des textes. Cependant, dans le processus d'argumentation un certain nombre d'objets ne peuvent émerger de cette façon. La petite «chanson » de la règle de trois (Spiesser 2003) en témoigne à mon sens. Il faudrait prendre au sérieux une descente dans les arcanes de la langue. Pourtant l'étude sur l'ensemble des textes ne me semble pas un angle d'approche pertinent : du fait de leur quantité, d'abord, mais aussi parce que ces textes, qui partagent une unité de langue, ne correspondent pas à des projets d'écriture équivalents (Lamassé 2104). Il faut donc morceler le travail et il me semble à ce propos que Nicolas Chuquet est le meilleur angle d'attaque afin de tester la méthode de Oaks-Bernard, notamment dans sa partie sur « les invencions de nombres » où il utilise la règle des premiers.

\section{Références}

Arrighi Gino, "Maestro umbro (sec. XIII), Livero de l'abbecho. Codice 2404 della Biblioteca Riccardiana di Firenze", dans Bollettino della deputazione di Storia patria per l'Umbria, 86 (1989), p. 5-140.

Bernard Alain et Proust Christine, dir., Scientific Sources and Teaching Contexts Throughout History : Problems and Perspectives, Springer, 2014.

Benoit Paul et Lamassé Stéphane, "Apposition et remotion : une spécificité française », dans De la Chine à l'Occitanie. Chemins entre arithmétique et algèbre, M. Charrier-Spiesser et M. Guillemot éd., Toulouse, Ed. du CIHSO, 2003, p. 1-14.

Beaujouan Guy, "The place of Nicolas Chuquet in a typology of fifteenth-century French arithmetics", dans Hay (Cynthia), éd., Mathematics from Manuscript to Print, 1300-1600, Oxford : Clarendon Press, 1988, p. 73-88.

Beaujouan Guy, «l'Enseignement de l'arithmétique élémentaire à l'université de Paris aux XIII et XIV siècle », Homenaje a Mollàs-Vallicrosa, vol. 1, Barcelone, 1954, p. 106 et seq.

Cassinet Jean, "Le manuscrit XXVI de Cesena : important maillon occitan de transmission de l'algorisme au 15ème siècle ». Bollettino Di Storia Delle Scienze Matematiche, 13(1993), p. 251-285.

Fery-Hue Françoise et Spiesser Maryvonne, « Le recueil mathématique de Cesena, Biblioteca Malatestiana, S.XXVI.6, au cœur du réseau franco-occitan des mathématiques pratiques du $\mathrm{XV}^{\mathrm{e}}$ siècle », dans Archives internationales d'Histoire des sciences, t. 158 (2007), p. 47-88. 
Genet Jean-Philippe, «Histoire, Informatique, Mesure », dans Histoire \& Mesure, 1986 vol. 1, pp. 7-18. Genet, Jean-Philippe et Zorzi, Andrea (éd.), Les Historiens et l'informatique. Un métier à réinventer, Rome, École française de Rome, 2011.

Genthilhomme Yves, «Contribution à une réflexion sur les locutions mathématiques », Cahiers du français contemporain, 2 (1995), p. 197-242

Gropp Harald, "Propositio de lupo et capra et fasciculo cauli - On the History of River - Crossing Problems" dans Charlemagne and his Heritage : 1200 Years of Civilization and Science in Europe: Karl der Grosse und sein Nachwirken: 1200 Jahre Kultur und Wissenschaft in Europa, Vol. 2: Mathematical Arts (eds. P. L. Butzer, H. Th. Jongen, W. Oberschelp), Turnhout, 1998, p. 31-41.

Hoyrup Jens, "Proportion in and around the Italian Abbacus Tradition" dans Physis, 46, 2009 (preprint disponible en ligne : http://www.akira.ruc.dk/ jensh /publications/ 2009\%7Bb\%7D_ \%27 Proportions $\% 27 \% 20$ in $\% 20$ and $\% 20$ around $\% 20$ the $\% 20$ Italian $\% 20 \mathrm{Abbacu}$ s\%20Tradition.pdf, consulté le 20/04/2015).

Labarthe Marie-Hélène, Premiéres arithmétiques imprimées des Espagne, Thèse de doctorat, sous la direction de Michel Guillemot, Toulouse 3, 2004, 3 vol. 889 p.

Lamassé Stéphane, "Relationships between French "practical arithmetics" and teaching?", dans Scientific Sources and Teaching Contexts Throughout History: Problems and Perspectives, (Bernard Alain et Proust Christine. ed.), Springer, 2014

Lamassé Stéphane, «Les traités d'arithmétique médiévale et la constitution d'une langue de spécialité », dans Sciences et langues au Moyen Âge : Actes de l'atelier franco-allemand, Paris, 27-30 janvier 2009, (Ducos J. dir), Heidelberg, 2012, p. 65-104

L'huillier Hervé, « Regards sur la formation progressive d'une langue pour les mathématiques dans l'Occident Médiéval », dans Comprendre et Maîtriser la nature : mélange d'histoire des sciences offerts à Guy Beaujouan, Droz, Genève, 1994, p. 541-555

Maryvonne Charrier Spiesser, Entre théorie et pratique : le compendy de la pratique des nombres de Barthélémy de Romans et Mathieu Préhode (1471): aspects, mathématiques, linguistiques et culturel, Thèse de doctorat sous la direction de Jean Dhombre, EHESS, Paris, 2001, 780 p.

Maryvonne Spiesser, Une arithmétique commerciale du $x v^{\mathrm{e}}$ siècle. Le Compendium de la Praticque des Nombres de Barthélémy de Romans, Turnhout, Brepols, 2003, 762 p.

McCarty Willard, Humanities Computing, Palgrave, 2014

Möhren Frankwalt, «Les débuts de l'écriture française de la géométrie au XIII ${ }^{\mathrm{e}}$ siècle », dans L'Ecriture du texte scientifique au Moyen Âge, Paris, PUPS, Cultures et civilisations médiévales, n³5, 2006, p. 93-116.

Roy Bruno, « Devinettes françaises du Moyen Âge », Cahiers d'Études Médiévales, 3, Paris, Vrin, 1977. Consultable partiellement sur internet : http://www.sites.univ-rennes2.fr/celam/cetm/ devinettes/devinettes.html (consulté le 30.10.2015).

Sesiano Jacques, Recreations Mathematiques Au Moyen-Âge. Lausanne, Presses polytechniques et universitaires romandes, 2014, $290 \mathrm{p}$.

Sesiano Jacques, « Une arithmétique médiévale en langue provençale », dans Centaurus, 1984, t. 27, p. $26-75$

Vera Sanford, The history and significance of certain standard problems in algebra, Thèse de doctorat, Columbia University, 1927.

Van Egmond W, "Types and Traditions of Mathematical Problems : A Challenge for Historians of Mathematics", dans M. Folkerts (ed.), Mathematische Probleme im Mittelalter. Der lateinische und arabische Sprachbereich, Wiesbaden, Otto Harrassowitz Verlag, 1996, p. 379-428

Vogel Kurt, Die Practica des algorismus ratisbonensis: ein rechenbuch des Benediktinerklosters St. Emmeram aus der Mitte des 15. Jahrhunderts nach den Handschriften der Münchner Staatsbibliothek und der Stifstsbibliothek st. Florian, Münich, 1954, 297 p. 